\title{
LIMIT THEOREMS FOR MEASURES ON NONMETRIZABLE LOCALLY COMPACT ABELIAN GROUPS $\left({ }^{1}\right)$
}

\author{
BY \\ DAVID C. BOSSARD
}

\begin{abstract}
In a recent book, Parthasarathy provides limit theorems for sums of independent random variables defined on a metrizable locally compact abelian group. These results make heavy use of the metric assumption. This paper consists of a reworking of certain results contained in Parthasarathy to see what can be done without the metric restriction. Among the topics considered are: necessary and sufficient conditions for a limit law to have an idempotent factor; the relationship between limits of compound Poisson laws and limits of sums of independent random variables; and a representation theorem for certain limit laws.
\end{abstract}

1. Introduction. A recent book by Parthasarathy, [1], includes a number of limit theorems for uniformly infinitesimal arrays of measures which are defined on metrizable locally compact abelian groups. These theorems provide generalizations of the classical theorems for sums of independent real-valued random variables which appear in the treatise of Gnedenko and Kolmogorov, [2].

The metric assumption is deeply involved in the proofs of the various results of [1] because of the extensive use of the Prokhorov Theorem [3] and the ShiftCompactness Theorem [4]. These theorems appear as Theorem II.6.7 and Theorem III.2.2 respectively in [1]. It can be shown (cf. [5]) that the assertions of these theorems are invalid on nonmetrizable locally compact abelian groups, even when the class of measures is restricted to regular Borel measures.

This paper consists of a reworking of certain results of [1] to see what can be done without the metric assumptions. In essence, it is a study of probability on locally compact abelian groups without using the Prokhorov Theorem. It turns out that the familiar limit theorems still hold, with slightly different statements concerning the assumptions, and, of course, with rather different proofs.

Three basic theorems are proved in this paper. The first basic theorem (Theorem 4.1) gives necessary and sufficient conditions for a limit law to have an idempotent factor. This theorem generalizes certain facts observed in [2] to hold for the real

Received by the editors June 8, 1970.

AMS 1969 subject classifications. Primary 6005, 6008, 6030.

Key words and phrases. Locally compact groups, nonmetrizable spaces, Prokhorov Theorem, conditional compactness of measures, probability measures, limit theorems, infinitesimal arrays, triangular arrays, infinitely divisible distributions, idempotent measures, characteristic functions, representation theorems, compound Poisson laws.

( $\left.{ }^{1}\right)$ Based on a doctoral dissertation [5] at Dartmouth College under the direction of Professor J. Lamperti; partial support was provided by a National Defense Education Act fellowship. Copyright (C) 1971, American Mathematical Society 
line. It is believed that this is a new result which is of interest for both metrizable and nonmetrizable locally compact abelian groups.

The second basic theorem (Theorem 5.1) shows the connection between limits of compound Poisson laws and convergence of row sums of infinitesimal arrays. This theorem is a modification of corresponding results in [1] and [2]; the principal change is that one of the derived consequences of the previous theorems is raised to the level of an assumption in the statement of the theorem itself. The assumption is related to the statement that "mass does not escape to infinity."

The third basic theorem (Theorem 7.1) is a representation theorem for certain limit laws. It corresponds to the well-known representation theorems for infinitely divisible laws of [1] and [2]. Because of the difficulties encountered on nonmetrizable groups, the theorem is stated in terms of a concept called "circle convergence." In the last section of the paper, circle convergence is shown to correspond to a generalized notion of convergence of the maximum term distributions.

2. Preliminaries. This section gives the notation, definitions, and other necessary background required for the following treatment. Most of the terminology used here is well known and so will be used without comment. However, the following definitions are required because of slight variations that exist in the literature. Frequent references to [6] are given for further details.

Throughout this paper, $G$ denotes a locally compact Hausdorff topological group, written multiplicatively with identity 1 . Such a group is a normal topological space $[6, \S 8.13]$ and is metrizable if and only if there exists a countable family of open sets with intersection $\{1\}[6, \S 8.5]$.

If $G$ is abelian, the characters form an abelian group which we denote by $\Gamma$ or $G^{\wedge}$. We denote characters by the letter $\gamma . \Gamma$ is a locally compact abelian topological group under the usual weak-* topology. If $G$ is compact, $\Gamma$ is discrete; if $G$ is discrete, $\Gamma$ is compact. The Duality Theorem asserts that $G$ is topologically isomorphic to $\Gamma^{\wedge}[6, \S 23.25]$.

A regular Borel measure is a nonnegative countably additive set function $m$ on the Borel sets of $G$, such that for every Borel set $E$,

$$
m(E)=\sup \{m(C): C \text { is compact and } C \subseteq E\} .
$$

A regular Borel measure is called finite if $m(G)<\infty$. The class of finite regular Borel measures on $G$ will be denoted by $M(G)$ and the subclass of $M(G)$ with total measure 1 will be denoted by $M_{1}(G)$. A measure in $M_{1}(G)$ which has unit mass on a single element $x$ will be denoted simply by " $x$." The measures in $M(G)$ arise in the theory of locally compact groups via the Riesz representation theorem.

If $\left\{m_{n}\right\}$ and $m$ are measures in $M(G)$, then $m_{n}$ converges weakly to $m$, written $m_{n} \Rightarrow m$, if

$$
\int f d m_{n} \rightarrow \int f d m
$$


for all bounded, continuous functions $f$. It is easy to verify by the Riesz theorem that the limit $m$ is unique if it exists.

The convolution $m * n$ of measures $m$ and $n$ from $M(G)$ is a measure in $M(G)$ defined for every Borel set $E$ by

$$
m * n(E)=\int m\left(E y^{-1}\right) d n(y)
$$

$[6, \S \S 19.8,19.11]$. If $x$ is a measure with unit mass at an element $x$ in $G$, then the convolution $m * x$ is a shift of $m$ by $x$.

In the following, the convolution of a sequence of measures $m_{1}, m_{2}, \ldots$ will be denoted by $\Pi^{*} m_{i}$. Thus $\prod_{i=1}^{* n} m_{i}(E)$ is the convolution $m_{1} * \cdots * m_{n}$ evaluated for the set $E$ whereas $\prod_{i=1}^{n} m_{i}(E)$ is the product $m_{1}(E) \cdots m_{n}(E)$. If $\zeta$ and $\xi$ are independent random variables with distributions $m$ and $n$, respectively, and if $\zeta+\xi$ is a random variable, then its distribution is given by $m * n$.

A measure $m$ is infinitely divisible if for each integer $n$ there is a measure $m_{n}$ such that $m=m_{n}^{* n}$ where $x^{* n}$ denotes the $n$-fold convolution of $x$.

Let $m$ be a measure in $M(G)$. The compound Poisson law $e(m)$ is defined by

$$
e(m)=e^{-m(G)} \sum_{j=0}^{\infty} m^{* j} / j !
$$

where $m^{* 0}$ is the measure with unit mass at the identity (also denoted by 1). As a formal definition, $e(m)$ defines a measure in $M_{1}(G)$, which is infinitely divisible. In fact, $e(m)=e(m / k)^{* k}$ where $m / k$ is the measure in $M(G)$ whose value for any set $E$ in $G$ is $(1 / k) m(E)$.

The Fourier transform (characteristic function) of a measure $m$ in $M(G)$ is the function $\hat{m}$ on $\Gamma$ defined for each $\gamma$ in $\Gamma$ by

$$
\hat{m}(\gamma)=\int \gamma(x) d m(x)
$$

The continuity theorem relates weak convergence of measures in $G$ to convergence of the Fourier transform. The following is the form that will be used here.

2.1. TheOREM (The CONTINUITy TheOREM). Let $G$ be a locally compact abelian group with character group $\Gamma$. Let $\left\{m_{n}\right\}$ and $m$ be measures in $M(G)$.

(i) If $m_{n} \Rightarrow m$, then $\hat{m}_{n}(\gamma) \rightarrow \hat{m}(\gamma)$ uniformly on every compact set in $\Gamma$.

(ii) If $\hat{m}_{n}(\gamma) \rightarrow s(\gamma)$ for each $\gamma$ in $\Gamma$, where $s$ is continuous and $s(1)<\infty$, then there is a measure $m$ in $M(G)$ such that $s=\hat{m}$ and $m_{n} \Rightarrow m$.

(iii) If $m$ and $m^{\prime}$ are measures in $M(G)$ and $\hat{m}(\gamma)=\hat{m}^{\prime}(\gamma)$ for all $\gamma$ in $\Gamma$, then $m=m^{\prime}$.

A class $A$ of measures (not necessarily with finite total measure) is tight if, given $\varepsilon>0$, there is a compact set $K_{\varepsilon}$ such that $m\left(G \sim K_{\varepsilon}\right)<\varepsilon$ for all $m$ in $A$. In particular, each measure in $M(G)$ is tight.

An idempotent measure is a measure $\lambda$ in $M_{1}(G)$ such that $\lambda=\lambda * \lambda$. The class of idempotent measures coincides with the class of normalized Haar measures of 
subgroups of $G$ (cf. [7, Theorem 5.2.1]). The class of Fourier transforms of idempotent measures coincides with the class of indicator functions of open-closed subgroups of $\Gamma$.

A symmetric measure is a measure such that $m(A)=m\left(A^{-1}\right)$ for every Borel set $A$. A measure is symmetric if and only if its Fourier transform is real valued. To each measure $m$ in $M(G)$ can be associated a symmetric measure $\bar{m} * m$ where $\bar{m}$ is defined by $\bar{m}(E)=m\left(E^{-1}\right)$ for every Borel set $E$ in $G$.

An array of measures is a matrix of measures of the form

$$
\left\{m_{n j}: j=1,2, \ldots, k_{n} ; n=1,2, \ldots\right\}
$$

where $k_{n} \rightarrow \infty$ as $n \rightarrow \infty$. An array is infinitesimal if, given $\varepsilon>0$ and any neighborhood $N$ of 1 , there is an integer $n_{\varepsilon, N}$ such that $m_{n j}(G \sim N)<\varepsilon$ for all $n>n_{\varepsilon, N}$ and $j=1, \ldots, k_{n}$. It is easy to check that if $\left\{m_{n j}\right\}$ is an infinitesimal array, then for any $\gamma$ in $\Gamma, \hat{m}_{n j}(\gamma) \rightarrow 1$ uniformly on $j$ as $n \rightarrow \infty$. When referring to arrays of measures, the notation $\sum_{j} m_{n j}, \prod_{j} m_{n j}$, and $\prod_{j}^{*} m_{n j}$ will always mean that the index $j$ runs from 1 to $k_{n}$; thus $\prod_{j}^{*} m_{n j}=m_{n 1} * m_{n 2} * \cdots * m_{n k_{n}}$, etc.

The preceding definitions and terminology are fairly standard in the literature. For the following development, it is convenient to add the following nonstandard term. A tight sequence $\left\{s_{n}\right\}$ of measures in $M(G)$ circle converges to a regular Borel measure $s$ (not necessarily in $M(G)$ ), written $s_{n} \stackrel{0}{\Rightarrow} s$ if $\int f d s_{n} \rightarrow \int f d s$ for every bounded continuous function $f$ which vanishes on some neighborhood of 1 . Circle convergence is similar to weak convergence except that things are permitted to blow up in the vicinity of the identity.

3. The centering function. In [1, Lemma IV.5.3], Parthasarathy proved the existence of a function $g(x, \gamma)$ on $G \times \Gamma$ which could be used in the task of "centering" the measures of an infinitesimal array so that the sums of independent random variables would converge to a limit. The development of this function as given in [1] is directly extendable to nonmetrizable locally compact abelian groups. Thus we will simply assert the existence of a function $g(x, \gamma)$ with stated properties and refer to [1] for the demonstration of its existence.

There exists for an arbitrary locally compact abelian group a function $g(x, \gamma)$ on $G \times \Gamma$ expressible in the form

$$
g(x, \gamma)=\sum_{\alpha \in I} g_{\alpha}(x) h_{\alpha}(\gamma)
$$

with the following properties:

(1) $\exp \left[-i \int g(x, \gamma) d m(x)\right]$ is a character on $\Gamma$ for each $m$ in $M(G)$,

(2) each $\gamma$ in $\Gamma$ is equal to $\exp [\operatorname{ig}(x, \gamma)]$ on some neighborhood $N_{\gamma}$ of 1 in $G$,

(3) $I$ is a specified index set (possibly infinite; countable if $G$ is metrizable),

(4) for each $\gamma$ in $\Gamma, h_{\alpha}(\gamma)$ is zero for all but finitely many $\alpha$,

(5) $g(x, \gamma)$ is continuous in $x$ and $\gamma$, and antisymmetric in $x$,

(6) for each $\alpha$ in $I, g_{\alpha}(x)$ is a bounded, continuous function with compact support, 
(7) for each $\alpha$ in $I$, there is a character $\gamma_{\alpha}$ such that $g\left(x, \gamma_{\alpha}\right)=g_{\alpha}(x)$.

Property (1) associates to each $m$ in $M_{1}(G)$ a centering element $x_{m}$ via the duality theorem:

$$
\gamma\left(x_{m}\right)=\exp \left[-i \int g(x, \gamma) d m(x)\right]
$$

Thus in the limit theorems we replace $m$ by the centered (shifted) distribution $m * x_{m}$. Property (2) is useful when $m$ has most of its mass on $N_{\gamma}$ (this happens, for example, if $m$ "approximates" the measure 1). For then

$$
\hat{m}(\gamma) \simeq \int_{N_{\gamma}} \gamma(x) d m(x)=\int_{N_{\gamma}} \exp [\operatorname{ig}(x, \gamma)] d m(x)
$$

and

$$
\hat{x}_{m}(\gamma)=\exp \left[-i \int g(x, \gamma) d m(x)\right]
$$

The similarity between these expressions is enough to make the limit theorems go through by using centered measures $m * x_{m}$.

Examples of the centering function are given in [1]. In particular

(i) For a discrete group, $g(x, \gamma) \equiv 0$,

(ii) For the real line, characters can be expressed in the form exp [ixt ]. Thus, let $g_{1}(x)$ be a continuous antisymmetric function bounded by 1 such that $g_{1}(x)=x$ for $x \in[-1,1]$ and vanishing outside $[-1-\varepsilon, 1+\varepsilon]$. Let $h_{1}(\exp [i x t])=t$. Then $g(x, \gamma)=g_{1}(x) h_{1}(\gamma)=x t$ for $x \in[-1,1]$. The integral $\int g_{1}(x) d m$ in property (1) plays the role of the truncated expectation in limit theorems on the real line (cf. [2, §25, Theorem 1]).

4. Idempotent factors in limit laws. The first basic theorem which we give addresses the question of whether a limit law has an idempotent factor. It is assumed that a centering function $g(x, \gamma)$ having the properties listed in $\S 3$ has been selected and is fixed for the following discussion.

4.1. THEOREM. Let $\left\{m_{n j}\right\}$ be an infinitesimal array of measures in $M_{1}(G)$ such that

$$
x_{n} * \prod_{j} * m_{n j} \Rightarrow m \text {. }
$$

Let $\left\{s_{n}\right\}$ be defined by

$$
s_{n}=\sum_{j} \bar{m}_{n j} * m_{n j}
$$

Then $m$ has an idempotent factor other than 1 if and only if one of the following conditions hold:

(i) $\lim \sup s_{n}(G \sim N)=\infty$ for some neighborhood $N$ of 1 , or

(ii) $\lim \int g_{\alpha}^{2}(x) d s_{n}(x)=\infty$ for some $\alpha$ in $I$. 
RemarK. This theorem is a refinement of [1, Theorem IV.4.3], in which conditions (i) and (ii) are included in a single expression (equation (1) below). Since the real line has no idempotent measures, this theorem applied to the real line asserts that if $x_{n} * \prod_{j}^{*} m_{n j}$ converges, then

(i') $\lim \sup s_{n}(G \sim N)<\infty$ for every neighborhood $N$ of 0 , and

(ii') $\lim \sup \int_{|x| \leqq 1} x^{2} d s_{n}(x)<\infty$.

These conditions are well known (cf. [2, pp. 110 and 118]).

Conditions (i) and (ii) are independent; that is, neither one implies the other. This is illustrated in the examples given in $\$ 9$.

The proof of this theorem requires two lemmas which we now give.

4.2. Lemma. A limit law $m$ has an idempotent factor (other than 1 ) if and only if $\hat{m}(\gamma)=0$ for some $\gamma$ in $\Gamma$.

Proof. Suppose $m=m_{1} * \lambda$ where $\lambda$ is an idempotent factor. Then $\hat{\lambda}$ is the indicator function of an open-closed subgroup $S$ in $\Gamma$. Pick $\gamma \in(\Gamma \sim S)$. Then

$$
\hat{m}(\gamma)=\hat{m}_{1}(\gamma) \hat{\lambda}(\gamma)=0 .
$$

Conversely, it can be shown that the set $H=\{\gamma: \hat{m}(\gamma) \neq 0\}$ is an open-closed subgroup of $\Gamma$. This is proved in Lemma IV.5.4 of [1] (the metric restriction is not used in the result). The indicator function of this subgroup is the Fourier transform of an idempotent factor of $m$. The lemma is proved.

4.3. LEMMA. Let $\left\{m_{n j}\right\}$ be an infinitesimal array of symmetric measures in $M_{1}(G)$ and let $\gamma$ be any character in $\Gamma$. Then

$$
\lim _{n \rightarrow \infty}\left|\prod_{j} \hat{m}_{n j}(\gamma)-\exp \left[-\sum_{j}\left(1-\hat{m}_{n j}(\gamma)\right)\right]\right|=0 .
$$

Proof. If $a_{1}, \ldots, a_{n}$ are real numbers such that $1-\varepsilon \leqq a_{j} \leqq 1$ for all $j$, then

$$
\prod_{j} a_{j}=\exp \left[-\sum_{j}\left(1-a_{j}\right)(1+\theta \varepsilon / 2)\right]
$$

for some $\theta$ with $|\theta|<1 /(1-\varepsilon)$. If $\varepsilon$ is sufficiently small, then we can approximate the product by the exponential. The lemma applies this fact to the double array of real numbers $\left\{\hat{m}_{n j}(\gamma)\right\}$. As $n \rightarrow \infty$, the numbers in the $n$th row are uniformly close to 1 . The lemma is proved.

Proof of Theorem 4.1. Using Lemma 4.3, the fact that $\bar{m}_{n j} * m_{n j}$ is symmetric and $\prod_{j}^{*} \bar{m}_{n j} * m_{n j} \Rightarrow \bar{m} * m$, it then follows that for each $\gamma$ in $\Gamma$,

i.e.,

$$
\lim _{n \rightarrow \infty}\left|\prod_{j}\left(\bar{m}_{n j} * m_{n j}\right)^{\wedge}(\gamma)-\exp \left[-\sum_{j}\left(1-\left(\bar{m}_{n j} * m_{n j}\right)^{\wedge}(\gamma)\right)\right]\right|=0,
$$

$$
\lim _{n \rightarrow \infty} \exp \left[\int(\operatorname{Re} \gamma(x)-1) d s_{n}\right]=\lim _{n \rightarrow \infty} \prod_{j}\left(\bar{m}_{n j} * m_{n j}\right)^{\wedge}(\gamma)=(\bar{m} * m)^{\wedge}(\gamma) .
$$


Suppose that $m$ has an idempotent factor. Then $(\bar{m} * m)^{\wedge}\left(\gamma_{0}\right)=0$ for some $\gamma_{0}$ in $\Gamma$. Suppose (i) does not hold. Then for any neighborhood $N$ of 1 in $G$,

$$
\int\left(\operatorname{Re} \gamma_{0}(x)-1\right) d s_{n}=\int_{N}\left(\operatorname{Re} \gamma_{0}(x)-1\right) d s_{n}+\int_{G \sim N}\left(\operatorname{Re} \gamma_{0}(x)-1\right) d s_{n} \rightarrow-\infty
$$

Hence

$$
\int_{N}\left(\operatorname{Re} \gamma_{0}(x)-1\right) d s_{n}(x) \rightarrow-\infty
$$

(Note. (1) is the expression contained in Theorem IV.4.3 of [1].)

By property (2) of $g(x, \gamma)$, there is a neighborhood $N_{\varepsilon}$ of 1 in $G$ such that $\left|g\left(x, \gamma_{0}\right)\right|<\varepsilon$ on $N_{\varepsilon}$ and $\gamma_{0}(x)=\exp \left[i g\left(x, \gamma_{0}\right)\right]$ for all $x$ in $N_{\varepsilon}$. Expanding $\operatorname{Re}\left(\gamma_{0}(x)\right)$ in a Taylor series at 1 , we have for all $x$ in $N_{\varepsilon}$

$$
\operatorname{Re}\left[\gamma_{0}(x)\right]-1=-(1 / 2) g^{2}\left(x, \gamma_{0}\right)\left(1+\theta_{x} \varepsilon^{2}\right) \text { where }\left|\theta_{x}\right|<1
$$

Hence

$$
\int g^{2}\left(x, \gamma_{0}\right) d s_{n}(x) \rightarrow \infty
$$

Now $g\left(x, \gamma_{0}\right)=\sum_{\alpha \in I} g_{\alpha}(x) h_{\alpha}\left(\gamma_{0}\right)$ and $h_{\alpha}\left(\gamma_{0}\right)=0$ for all but finitely many $\alpha$. Hence from

$$
\int g^{2}\left(x, \gamma_{0}\right) d s_{n}(x)=\sum_{\alpha, \beta \in I} h_{\alpha}\left(\gamma_{0}\right) h_{\beta}\left(\gamma_{0}\right) \int g_{\alpha}(x) g_{\beta}(x) d s_{n}(x) \rightarrow \infty,
$$

it follows that, for some $\alpha, \beta$ in $I$,

$$
\lim \sup \int g_{\alpha}(x) g_{\beta}(x) d s_{n}(x)=\infty .
$$

The Hölder inequality then yields the fact that for some $\alpha$

$$
\lim \sup \int g_{\alpha}^{2}(x) d s_{n}(x)=\infty
$$

This is almost (ii). The reader can check the additional point that in fact $(\bar{m} * m)^{\wedge}\left(\gamma_{\alpha}\right)$ $=0$ where $\gamma_{\alpha}$ is the character for which $g\left(x, \gamma_{\alpha}\right)=g_{\alpha}(x)$. This implies that the limit and not just the lim sup holds in (ii).

To prove the converse half of the theorem, observe that if (ii) holds and (i) does not, then $\hat{m}\left(\gamma_{\alpha}\right)=0$ and so $m$ has an idempotent factor. Thus the only case to prove is to show that (i) implies that $m$ has an idempotent factor. We will prove this assertion in several steps.

Case A. $G$ is a compact group. Let $N$ be a neighborhood of 1 in $G$ such that $\lim \sup s_{n}(G \sim N)=\infty$. Now members of $\Gamma$ separate points of $G[6, \S 22.27]$, i.e., given $x \neq 1$, there is a character $\gamma_{x}$ such that $\gamma_{x}(x) \neq 1$. Since $1-\operatorname{Re} \gamma_{x}(y)$ is a nonnegative continuous function of $y$ on $G$, there is a neighborhood $N_{x}$ of $x$ such that

$$
1-\operatorname{Re} \gamma_{x}(y)>\left(1-\operatorname{Re} \gamma_{x}(x)\right) / 2
$$


for all $y$ in $N_{x}$. Cover $G$ by $N$ together with the sets $N_{x}$ where $x$ runs through $G \sim N$. Reduce to a finite subcover: $N, N_{1}, \ldots, N_{j}$ where $N_{i}=N_{x_{i}}$ for some $x_{i}$. Since lim sup $s_{n}(G \sim N)=\infty$ and since $s_{n}(G \sim N) \leqq \sum_{i=1}^{j} s_{n}\left(N_{i}\right)$ it follows that lim sup $s_{n}\left(N_{i}\right)$ $=\infty$ for some $i$. Let $\gamma^{\prime}$ denote the character corresponding to this neighborhood. Then

$$
-\int\left(\operatorname{Re} \gamma^{\prime}(x)-1\right) d s_{n}(x) \geqq-\int_{N_{i}}\left(\operatorname{Re} \gamma^{\prime}(x)-1\right) d s_{n}(x) \geqq\left(1-\operatorname{Re} \gamma_{x_{i}}\left(x_{i}\right)\right) s_{n}\left(N_{i}\right) / 2
$$

and so in fact

$$
\limsup _{n \rightarrow \infty}\left[-\int\left(\operatorname{Re} \gamma^{\prime}(x)-1\right) d s_{n}(x)\right]=\infty
$$

and so $\hat{m}\left(\gamma^{\prime}\right)=0$ so that $m$ has an idempotent factor.

Case B. G is a metrizable group. This case is proved in [1] as the first step in the proof of Theorem IV.4.3. Observe that $e\left(s_{n}\right) \Rightarrow \bar{m} * m$.

Case C. The general case. $G$ has an open compact subgroup $C$. If

$$
\lim \sup s_{n}(G \sim C)<\infty,
$$

then we proceed as in Case A. If $\lim \sup s_{n}(G \sim C)=\infty$, then note that there is convergence on the discrete group $G / C$ and the limit law has an idempotent factor (since $G / C$ is metrizable we are in Case B). Let $\pi_{0}: G \rightarrow G / C$ be the canonical map and let $\gamma_{0}$ be a character on $(G / C)^{\wedge}$ such that $\hat{m}_{0}\left(\gamma_{0}\right)=0$. Let $\gamma$ be the character on $\Gamma$ defined by $\gamma(x)=\gamma_{0}\left(\pi_{0} x\right)$. Then $\hat{m}(\gamma)=0$. Thus $m$ has an idempotent factor. The theorem is proved.

5. A theorem on limits. We will now give a result which relates certain limit laws with limits of shifts of compound Poisson laws. Our result corresponds to Theorem IV.5.1 of [1]. Note that assumption (i) is a consequence of the statement of the theorem in [1], appearing in equation (IV.5.14).

5.1. TheOREM. Let $G$ be a locally compact abelian group and let $\left\{m_{n j}\right\}$ be an infinitesimal array of measures such that

$$
\limsup _{n \rightarrow \infty} \sum_{j} m_{n j}(G \sim N)<\infty
$$

for each neighborhood $N$ of 1 . Then for each $\gamma$ in $\Gamma$,

$$
\lim _{n \rightarrow \infty}\left|\sum_{j}\left(m_{n j} * x_{n j}\right)^{\wedge}(\gamma)-\hat{e}\left(\sum_{j} m_{n j} * x_{n j}\right)(\gamma)\right|=0,
$$

where $x_{n j}$ is defined by

$$
\hat{x}_{n j}(\gamma)=\exp \left[-i \int g(x, \gamma) d m_{n j}(x)\right] .
$$

This theorem has the following corollary. 
5.2. COROllary. Let $\left\{m_{n j}\right\}$ be an infinitesimal array such that

$$
x_{n} * \prod_{j}^{*} m_{n j} \Rightarrow m
$$

where $m$ has no idempotent factor. Then there is a sequence of elements $\left\{z_{n}\right\}$ in $G$ such that

$$
e\left(\sum_{j} m_{n j} * x_{n j}\right) * z_{n} \Rightarrow m .
$$

Thus every limit law without idempotent factors is the limit of shifts of a sequence of compound Poisson laws.

Proof of the theorem. The definition of $x_{n j}$ makes sense because of property (1) of the special function $g(x, \gamma)$. For large $n$,

$$
\left|1-\left(m_{n j} * x_{n j}\right)^{\wedge}(\gamma)\right|<\varepsilon
$$

uniformly on $j$, and so for large $n$,

$$
\prod_{j}\left(m_{n j} * x_{n j}\right)^{\wedge}(\gamma)=\exp \left[-\left(1+\theta_{n} \varepsilon\right) \sum_{j} \int(1-\gamma(x)) d m_{n j} * x_{n j}\right]
$$

where $\left|\theta_{n}\right|<1$. Note that

$$
\hat{e}\left(\sum_{j} m_{n j} * x_{n j}\right)(\gamma)=\exp \left[-\sum_{j} \int(1-\gamma(x)) d m_{n j} * x_{n j}\right] .
$$

Suppose the conclusion of the theorem is not true. Then for some $\gamma$ in $\Gamma$ and $\delta>0$ and a subsequence of $n$ (which we will denote by $n$ itself), we have

$$
\left|\prod_{j}\left(m_{n j} * x_{n j}\right)^{\wedge}(\gamma)-\hat{e}\left(\sum_{j} m_{n j} * x_{n j}\right)(\gamma)\right|>\delta>0 \text { for all } n \text {. }
$$

Pick a neighborhood $N$ of 1 sufficiently small that

$$
\gamma(x)=\exp [i g(x, \gamma)], \quad g(x y, \gamma)=g(x, \gamma)+g(y, \gamma), \quad|g(x y, \gamma)|<\varepsilon
$$

for all $x, y$ in $N$. Then

$$
\int(1-\gamma(x)) d m_{n j} * x_{n j}=\int_{N}\left(1-\gamma\left(x x_{n j}\right)\right) d m_{n j}+\int_{G \sim N}\left(1-\gamma\left(x x_{n j}\right)\right) d m_{n j} .
$$

On $N$,

$$
\begin{aligned}
\left(1-\gamma\left(x x_{n j}\right)\right) & =1-\exp \left[i g\left(x x_{n j}, \gamma\right)\right] \\
& =-i g\left(x x_{n j}, \gamma\right)+g^{2}\left(x x_{n j}, \gamma\right)(1+\theta \varepsilon) / 2 \text { for }|\theta|<1
\end{aligned}
$$

Now for large $n$,

$$
g\left(x x_{n j}, \gamma\right)=g(x, \gamma)+g\left(x_{n j}, \gamma\right)=g(x, \gamma)-\int g(x, \gamma) d m_{n j}
$$


so

$$
\begin{aligned}
i \int_{N} g\left(x x_{n j}, \gamma\right) d m_{n j} & =i \int_{N} g(x, \gamma) d m_{n j}-i m_{n j}(N) \int g(x, \gamma) d m_{n j} \\
& =i\left(1-m_{n j}(N)\right) \int g(x, \gamma) d m_{n j}-i \int_{G \sim N} g(x, \gamma) d m_{n j}
\end{aligned}
$$

Combining these results we obtain

$$
\begin{array}{rl}
\sum_{j} \int(1-\gamma(x)) d m_{n j} & * x_{n j} \\
= & -i \sum_{j}\left(1-m_{n j}(N)\right) \int g(x, \gamma) d m_{n j}+i \sum_{j} \int_{G \sim N} g(x, \gamma) d m_{n j} \\
& +\left[\left(1+\theta^{\prime \prime} \varepsilon\right) / 2\right] \sum_{j} \int g^{2}(x, \gamma) d m_{n j} * x_{n j} \\
& -\left[\left(1+\theta^{\prime} \varepsilon\right) / 2\right] \sum_{j} \int_{G \sim N} g^{2}\left(x x_{n j}, \gamma\right) d m_{n j} \\
& +\sum_{j} \int_{G \sim N}\left(1-\gamma\left(x x_{n j}\right)\right) d m_{n j} .
\end{array}
$$

The first term on the right in (5) tends to 0 as $n \rightarrow \infty$ and all but the third term are uniformly bounded on $n$, by assumption. But if (4) holds, then the third term is also uniformly bounded, for otherwise for some subsequence, the magnitude of the sum in (2) and (3) becomes infinite according to (5), i.e., both terms in (4) tend to zero in magnitude for this subsequence, contradicting (4). Thus, if (4) holds the sum

$$
\sum_{j} \int(1-\gamma(x)) d m_{n j} * x_{n j}<M<\infty \text { for all } n .
$$

But then (2) and (3) lead to the conclusion that

$$
\begin{aligned}
& \prod_{j}\left(m_{n j} * x_{n j}\right) \wedge(\gamma) / \hat{e}\left(\sum_{j} m_{n j} * x_{n j}\right)(\gamma) \\
&=\exp \left[-\theta_{n} \varepsilon \sum_{j} \int(1-\gamma(x)) d m_{n j} * x_{n j}\right] \rightarrow 1,
\end{aligned}
$$

since $\varepsilon$ is arbitrary, which again contradicts (4). Hence (4) cannot hold, and the theorem is established.

Proof of the corollary. We need only check that

$$
\lim \sup \sum_{j} m_{n j}(G \sim N)<\infty
$$

for every neighborhood $N$ of 1 , for then the result follows easily from Theorem 5.1. But $\bar{m} * m$ has no idempotent factor and so from Theorem 4.1, we have

$$
\lim \sup \sum_{j} \bar{m}_{n j} * m_{n j}(G \sim N)<\infty
$$


for every neighborhood $N$ of 1 . Pick $N=U^{2}$. Then

$$
\begin{aligned}
\bar{m}_{n j} * m_{n j}(G \sim U) & =\int m_{n j}\left(G \sim U y^{-1}\right) d \bar{m}_{n j} \geqq \int_{U} m_{n j}\left(G \sim U y^{-1}\right) d \bar{m}_{n j} \\
& \geqq \inf _{y \in U} m_{n j}\left(G \sim U y^{-1}\right) \bar{m}_{n j}(U) \geqq m_{n j}\left(G \sim U^{2}\right) \bar{m}_{n j}(U) \\
& \geqq m_{n j}(G \sim N) \bar{m}_{n j}(U) .
\end{aligned}
$$

It follows from this that (6) holds. End of proof.

If the group is metrizable, it is possible to give a more precise specification of what the limit laws are: they are the infinitely divisible laws defined in the sense of Parthasarathy, [1]. This follows from an easy lemma which uses the shift-compactness theorem: limits of shifts of infinitely divisible laws are again infinitely divisible [1, Theorem IV.4.1].

6. Weak convergence implies circle convergence? In this and the following section, the relation of weak convergence and circle convergence will be considered. The results of this section correspond to [2, §25], which can be interpreted as relating these two types of convergence.

6.1. TheOREM. Let $G$ be a locally compact abelian group and let $\left\{m_{n j}\right\}$ be an infinitesimal array of measures such that

Then $\left\{\sum_{j} m_{n j}\right\}$ is tight.

$$
x_{n} * \prod_{j}^{*} m_{n j} \Rightarrow m \text {. }
$$

6.2. Corollary. Let $G$ be a metrizable locally compact abelian group and let $\left\{m_{n j}\right\}$ be an infinitesimal array of measures such that

$$
x_{n} * \prod_{j}^{*} m_{n j} \Rightarrow m
$$

where $m$ has no idempotent factor. Then there is a subsequence of $\left\{\sum_{j} m_{n j}\right\}$ which circle converges.

Proof of the theorem. It is enough to show the following: if $\left\{m_{n j}\right\}$ is an infinitesimal array of symmetric measures and

$$
\prod_{j}^{*} m_{n j} \Rightarrow m
$$

then $\left\{\sum_{j} m_{n j}\right\}$ is tight. From this it follows that if

$$
\prod_{j}^{*} \bar{m}_{n j} * m_{n j} \Rightarrow \bar{m} * m
$$

then $\left\{\sum_{j} \bar{m}_{n j} * m_{n j}\right\}$ is tight and from this we conclude that $\left\{\sum_{j} m_{n j}\right\}$ is tight. For pick $\varepsilon>0$ and let $U$ be a symmetric neighborhood of 1 such that $U^{-}$is compact and

$$
\sum_{j} \bar{m}_{n j} * m_{n j}(G \sim U)<\varepsilon / 2
$$


for all $n$. Then $\left(U^{2}\right)^{-}$is compact and for sufficiently large $n$

$$
\sum_{j} m_{n j}\left(G \sim U^{2}\right)<\varepsilon .
$$

This follows from an argument given in the proof of Corollary 5.2.

By [1, Lemma IV.5.4], the limit measure $m$ has a maximal idempotent factor; namely, the normalized Haar measure whose support is the compact subgroup $C$ such that $C^{\perp}=\{\gamma: \hat{m}(\gamma) \neq 0\}$. If $\pi_{0}: G \rightarrow G / C$ is the canonical map, then

$$
\prod_{j}^{*}\left(m_{n j}\right)_{0} \Rightarrow(m)_{0} \text {. }
$$

Further, $(m)_{0}$ has no idempotent factor. Thus if $N$ is any neighborhood of 1 with compact closure, then

$$
\lim \sup \sum_{j} m_{n j}(G \sim N C)<\infty
$$

and so we conclude that there is a neighborhood $R$ of 1 in $G$ with compact closure (namely $N C$ ) with lim sup $s_{n}(G \sim R)<\infty$ where $s_{n}=\sum_{j} m_{n j}$.

It only remains to show that $\left\{\left.s_{n}\right|_{G \sim R}\right\}$ is tight, for then obviously $\left\{s_{n}\right\}$ itself is tight. If $G$ is metrizable, then the shift-compactness theorem holds. Now

$$
e\left(s_{n}\right)=e\left(\left.s_{n}\right|_{R}\right) * e\left(\left.s_{n}\right|_{G \sim R}\right) \Rightarrow m .
$$

Thus $\left\{e\left(\left.s_{n}\right|_{G \sim R}\right)\right\}$ is shift-compact and hence $\left\{e\left(\left.2 s_{n}\right|_{G \sim R}\right)\right\}$ is conditionally compact by the relation

$$
\left\{e\left(\left.2 s_{n}\right|_{G \sim R}\right)\right\}=\bar{e}\left(\left.s_{n}\right|_{G \sim R}\right) * e\left(\left.s_{n}\right|_{G \sim R}\right) .
$$

Given $\varepsilon>0$, there is a compact set $K_{\varepsilon}$ such that

$\varepsilon>e\left(\left.2 s_{n}\right|_{G \sim R}\right)\left(G \sim K_{\varepsilon}\right) \geqq\left.\exp \left[-2 s_{n}(G \sim R)\right] s_{n}\right|_{G \sim R}\left(G \sim K_{\varepsilon}\right) \geqq\left. M s_{n}\right|_{G \sim R}\left(G \sim K_{\varepsilon}\right)$

for some constant $M$. Thus $\left\{\left.s_{n}\right|_{G \sim R}\right\}$ is tight.

In the nonmetrizable case, $G$ has an open compact subgroup $C$. Since $G / C$ is discrete, the induced set of measures $\left\{\left(s_{n}\right)_{0}\right\}$ on $G / C$ is tight. But tightness on $G / C$ means that all but $\varepsilon$ of the mass of the measures $s_{n}$ lies on a finite number of cosets of $C$ in $G$, and so $\left\{s_{n}\right\}$ is tight. End of proof.

Proof of the corollary. Let $\left\{N_{i}\right\}$ be a countable basis at the identity. Use Theorem 5.1, the Prokhorov Theorem, and a diagonalization procedure to find a subsequence of $\left\{\sum_{j} m_{n j}\right\}$ which converges weakly when restricted to $G \sim N_{i}$ for each $i$. Then this subsequence circle converges. End of proof.

7. Circle convergence implies weak convergence? Now we will investigate the conditions under which $x_{n} * \prod_{j}^{*} m_{n j} \Rightarrow m$ (where $m$ has no idempotent factor) when it is assumed that $\sum_{j} m_{n j} \stackrel{0}{\Rightarrow} s$. It is perhaps worth emphasizing that the limit laws without idempotent factors are precisely those with the property that $\bar{m} * m$ is an infinitely divisible measure in the sense of Grenander, [7]. We will follow the statement of the theorem with a corollary which gives a valuable side result. 
7.1. TheOREM. Let $G$ be a locally compact abelian group and let $\left\{m_{n j}\right\}$ be an infinitesimal array of measures in $M_{1}(G)$ such that

$$
\sum_{j} m_{n j} \stackrel{0}{\longrightarrow} s
$$

where $s$ is a regular Borel measure such that $s(G \sim N)<\infty$ for all neighborhoods $N$ of 1 . Then there is a sequence $\left\{z_{n}\right\}$ of elements in $G$ such that

$$
z_{n} * \prod_{j}^{*} m_{n j} \Rightarrow m
$$

if and only if there is a measure $m^{\prime}$ such that

$$
e\left(\sum_{j} m_{n j} * x_{n j}\right) \Rightarrow m^{\prime}
$$

where $x_{n j}$ is defined by its characteristic function

$$
\hat{x}_{n j}(\gamma)=\exp \left[-i \int g(x, \gamma) d m_{n j}(x)\right] .
$$

In this case, there is an element $z$ in $G$ such that

$$
m=m^{\prime} * z
$$

The limit law $m^{\prime}$ is infinitely divisible and its characteristic function is given by

where

$$
m^{\prime}(\gamma)=\exp [-\phi(\gamma)] \exp \left[-\int\left(1-\gamma(x)+i g(x, \gamma)-g^{2}(x, \gamma) / 2\right) d s\right]
$$

$$
\phi(\gamma)=(1 / 2) \lim \sum_{j} \int g^{2}(x, \gamma) d\left(m_{n j} * x_{n j}\right)
$$

(the limit exists for each $\gamma$ in $\Gamma$ ).

7.2. Corollary. Every limit law $m$ without idempotent factors such that it is the limit of some circle-convergent array is the shift of an infinitely divisible law whose characteristic function is given by (i) above.

Proof of the theorem. The functions $g^{2}\left(x x_{n j}, \gamma\right)$ and $\gamma\left(x x_{n j}\right)$ converge uniformly on compact sets to the functions $g^{2}(x, \gamma)$ and $\gamma(x)$ as $n \rightarrow \infty$. If $G \sim N$ is a continuity set of $s$, then it follows from circle convergence and the tightness of $s$ that each of the sums on the right side of equation (5) of $\$ 5$ converge as follows:

$$
\begin{aligned}
\lim \sum_{j}\left(1-m_{n j}(N)\right) \int g(x, \gamma) d m_{n j} & =0 \\
\lim \sum_{j} \int_{G \sim N} g(x, \gamma) d m_{n j} & =\int_{G \sim N} g(x, \gamma) d s, \\
\lim \sum_{j} \int_{G \sim N} g^{2}\left(x x_{n j}, \gamma\right) d m_{n j} & =\int_{G \sim N} g^{2}(x, \gamma) d s \\
\lim \sum_{j} \int_{G \sim N}\left(1-\gamma\left(x x_{n j}\right)\right) d m_{n j} & =\int_{G \sim N}(1-\gamma(x)) d s .
\end{aligned}
$$


Hence we have the following, where we assume for convenience, that the remaining limits in (5) occur

$$
\begin{aligned}
\lim \sum_{j} \int(1-\gamma(x)) d m_{n j} * x_{n j}= & \int_{G \sim N}\left[1-\gamma(x)+i g(x, \gamma)-\left(1+\theta^{\prime} \varepsilon\right) g^{2}(x, \gamma) / 2\right] d s \\
& +(1 / 2)\left(1+\theta^{\prime \prime} \varepsilon\right) \lim \sum_{j} \int g^{2}(x, \gamma) d m_{n j} * x_{n j} .
\end{aligned}
$$

If $e\left(\sum_{j} m_{n j} * x_{n j}\right) \Rightarrow m^{\prime}$, then $z_{n} * \prod_{j}^{*} m_{n j} \Rightarrow m^{\prime}$ with $z_{n}=\prod_{j}^{*} x_{n j}$ by Theorem 5.1 and so the only thing to prove in the first part of the theorem is that if $m$ exists, then $m^{\prime}$ exists. Thus suppose that $z_{n} * \prod_{j}^{*} m_{n j} \Rightarrow m$ for some sequence $\left\{z_{n}\right\}$. Then for each $\gamma$ in $\Gamma$,

$$
\left|\left[\prod_{j} \hat{m}_{n j}(\gamma)\right] \hat{z}_{n}(\gamma)\right|=\left|\prod_{j}\left(m_{n j} * x_{n j}\right)^{\wedge}(\gamma)\right| \rightarrow|\hat{m}(\gamma)| .
$$

If $|\hat{m}(\gamma)|=0$, then from (2) we have

$$
\lim \operatorname{Re}\left[\sum_{j} \int(1-\gamma(x)) d m_{n j} * x_{n j}\right]=\infty
$$

and so from (7) we see that

$$
\lim \left[\sum_{j} \int g^{2}(x, \gamma) d m_{n j} * x_{n j}\right]=\infty=\phi(\gamma)
$$

and $\hat{m}^{\prime}(\gamma)=0$ in equation (i) (we will agree to write $\infty \cdot 0=0$ in this equation).

If $|\hat{m}(\gamma)| \neq 0$, then again from (2) we have

so, from (7),

$$
\lim \sup \operatorname{Re}\left[\sum_{j} \int(1-\gamma(x)) d m_{n j} * x_{n j}\right]<\infty,
$$

$$
\lim \sup \sum_{j} \int g^{2}(x, \gamma) d m_{n j} * x_{n j}<\infty .
$$

The integrand of the integral over $G \sim N$ on the right of (7) is uniformly bounded for all neighborhoods $N$. Thus we can let $\varepsilon \rightarrow 0$ and integrate over all of $G$, with the result

$$
\begin{aligned}
\lim \sum_{j} \int(1-\gamma(x)) d m_{n j} * x_{n j}= & \int\left(1-\gamma(x)+i g(x, \gamma)-g^{2}(x, \gamma) / 2\right) d s \\
& +(1 / 2) \lim \sum_{j} \int g^{2}(x, \gamma) d m_{n j} * x_{n j}
\end{aligned}
$$

If we let $\varepsilon \rightarrow 0$ in (2), we see that the real part of (8) exists as a limit since it yields the magnitude of $\hat{m}(\gamma)$, so in fact the limit on the right of (8) does exist and hence the left-hand limit exists. Thus $\hat{e}\left(\sum m_{n j} * x_{n j}\right)$ tends pointwise to a limit which is 
given by (i). It only remains to show that this limit is a continuous function on $\Gamma$ and then we will have established the first part of the theorem.

$\hat{m}^{\prime}(\gamma)=0$ if and only if $\hat{m}(\gamma)=0$ and so the subset of $\Gamma$ on which $\hat{m}^{\prime}(\gamma)$ is nonzero is an open and closed subgroup. Thus we need only check continuity at any $\gamma_{0}$ for which $\hat{m}^{\prime}\left(\gamma_{0}\right)$ is nonzero. The real part of (7) tends to a continuous limit since it gives the magnitude of $\hat{m}(\gamma)$. The continuity of $\operatorname{Re} \int_{G \sim N}(\quad) d s$ at $\gamma_{0}$ is trivial and so it follows from (7) that

$$
\lim \sum_{j} \int g^{2}(x, \gamma) d m_{n j} * x_{n j}
$$

is continuous. Hence $\phi(\gamma)$ is continuous. Also from (7) we see that

$$
\operatorname{Im}\left[\lim \sum_{j} \int(1-\gamma(x)) d m_{n j} * x_{n j}\right]
$$

is continuous and so (8) shows that the integral part of $\hat{m}^{\prime}(\gamma)$ is continuous. Hence $\hat{m}^{\prime}(\gamma)$ is a continuous function and the continuity theorem then asserts it is the Fourier transform of a measure $m^{\prime}$.

The infinite divisibility of $m^{\prime}$ follows from the fact that $\hat{e}\left(\sum m_{n j} * x_{n j} / k\right)$ tends to the $k$ th principle root of $\hat{m}^{\prime}(\gamma)$ which is also continuous.

The final task is to show that there is an element $z$ such that $m=m^{\prime} * z$. Now

$$
a_{n}=\prod_{j}^{*} m_{n j} * x_{n j} \Rightarrow m^{\prime} \text { and } a_{n} * y_{n} \Rightarrow m \text {. }
$$

Let $H=\left\{\gamma: \hat{m}^{\prime}(\gamma) \neq 0\right\}$. Then for each $\gamma$ in $H$,

$$
\gamma\left(y_{n}\right)=\hat{y}_{n}(\gamma)=\frac{\hat{a}_{n}(\gamma) \hat{y}_{n}(\gamma)}{\hat{a}_{n}(\gamma)} \rightarrow \frac{\hat{m}(\gamma)}{\hat{m}^{\prime}(\gamma)}=f(\gamma)
$$

and $f(\gamma)$ is continuous on $H$ since $\hat{m}$ and $\hat{m}^{\prime}$ are. Hence $f(\gamma)$ is a character on $H$ since it is the continuous limit of characters on $H$. Let $\gamma(z)$ be any character which extends $f(\gamma)$ to a character on $\Gamma$. Then for each $\gamma$ in $H, \hat{y}_{n}(\gamma) \rightarrow \hat{z}(\gamma)$ and so

$$
\left(a_{n} * y_{n}\right)^{\wedge}(\gamma) \rightarrow \hat{m}^{\prime}(\gamma) \hat{z}(\gamma) \text { for all } \gamma \text {. }
$$

Thus $m=m^{\prime} * z$. The theorem is proved.

A normal law is a limit of an infinitesimal array which circle converges to $s=0$. Thus if $m$ is a normal law, then its Fourier transform is of the form

$$
\hat{m}(\gamma)=\gamma(z) \exp [-\phi(\gamma)] .
$$

7.3. THEOREM. Every normal law without idempotent factors has a Fourier transform of the form

$$
\hat{m}(\gamma)=\gamma(z) \exp \left[-\sum_{\alpha, \beta \in I} c_{\alpha \beta} h_{\alpha}(\gamma) h_{\beta}(\gamma)\right]
$$

where $\left|c_{\alpha \beta}\right|<\infty$ for each $\alpha, \beta$. 
Proof. For each $\gamma, h_{\alpha}(\gamma)$ is nonzero for only finitely many $\alpha \in I$. Hence

Let

$$
\begin{aligned}
\sum_{j} \int g^{2}(x, \gamma) d m_{n j} * x_{n j} & =\sum_{j} \int \sum_{\alpha, \beta \in I} g_{\alpha}(x) g_{\beta}(x) h_{\alpha}(\gamma) h_{\beta}(\gamma) d m_{n j} * x_{n j} \\
& =\sum_{\alpha, \beta} h_{\alpha}(\gamma) h_{\beta}(\gamma) \sum_{j} \int g_{\alpha}(x) g_{\beta}(x) d m_{n j} * x_{n j} .
\end{aligned}
$$

$$
c_{\alpha \beta}=\lim \sum_{j} \int g_{\alpha}(x) g_{\beta}(x) d m_{n j} * x_{n j} / 2
$$

(the limit exists and is finite). Then the theorem follows from Theorem 7.1.

Much more can be said of the normal laws. We refer the reader to [1] for more details.

8. What is circle convergence? Let $\left\{m_{n j}\right\}$ be an array of random variables in $M_{1}(G)$, and let $f$ be a continuous function on $G$ with $f(1)=0$. Define real-valued functions $F_{f, n}(t)$ by

$$
F_{f, n}(t)=\prod_{j} m_{n j}(\{x:|f(x)| \leqq t\}) .
$$

This defines a proper distribution function on the real line called the $n$th maximum term distribution corresponding to $f$. If the measures $m_{n 1}, \ldots, m_{n k_{n}}$ are distributions of independent random variables, then $F_{f, n}(t)$ is the probability that the maximum value of $|f(x)|$ does not exceed $t$. If, for every continuous function $f$ with $f(1)=0$, the maximum term distribution converges to a proper distribution function as $n \rightarrow \infty$, then we say that the maximum term distributions converge.

We will now show that for infinitesimal arrays, circle convergence is equivalent to convergence of the maximum term distributions. Note that $G$ need not be abelian.

8.1. TheOREM. Let $G$ be a locally compact group and let $\left\{m_{n j}\right\} \subseteq M_{1}(G)$ be an infinitesimal array. Then the maximum term distributions converge if and only if there exists a tight regular Borel measure s such that

$$
s_{n}=\sum_{j} m_{n j} \stackrel{0}{\longrightarrow} s .
$$

Proof. Let $f$ be a continuous function such that $f(1)=0$ and let $F_{f, n}(t)$ be the maximum term distribution for $f$, computed for the $n$th row of the array. Then

$$
F_{f, n}(t) \sim \exp \left[-s_{n}\{x:|f(x)|>t\}\right]
$$

and so $F_{f, n}(t) \rightarrow F_{f}(t)$ if and only if

$$
s_{n}\{x:|f(x)|>t\} \rightarrow-\log F_{f}(t) .
$$

Suppose that the maximum term distributions converge. Then for every continuous function $f$ with $f(1)=0, F_{f, n}(t) \rightarrow F_{f}(t)$ a.e., where $F_{f}(t)$ is a proper distribution function. 
First we note that the set $\left\{s_{n}\right\}$ is tight. For suppose not. Let $N$ be a neighborhood of 1 with compact closure. Obtain a sequence of compact sets $\left\{K_{i}\right\}$ and $\varepsilon>0$ such that

(i) $K_{0}=\bar{N} \subset K_{1} \subseteq K_{1} N \subset K_{2} \subseteq K_{2} N \subset \ldots \subset K_{i} \subseteq K_{i} N \subset \ldots$,

(ii) $K_{i} \neq K_{i+1}$ for each $i=0,1,2, \ldots$, and

(iii) for each $i$ there is an integer $n_{i}$ such that $s_{n}\left(G \sim K_{i}\right)>\varepsilon$.

Such a sequence can be constructed by using the fact that each $s_{n}$ is tight, but $\left\{s_{n}\right\}$ is not tight. Using Urysohn's lemma and observing that $G$ is a normal topological space, it is possible to find a continuous function $f$ on $G$ such that

and

$$
f(x) \leqq i \text { for all } x \text { in } K_{i}, i=0,1,2, \ldots,
$$

$$
f(x)=i \text { for all } x \text { in } K_{i} \sim K_{i-1} N, i=1,2, \ldots
$$

By assumption, $F_{f, n}(t) \rightarrow F_{f}(t)$ a.e. and $F_{f}$ is proper. Thus given $\varepsilon>0$, there is an integer $t_{\varepsilon}$ such that $F_{f, n}\left(t_{\varepsilon}\right) \rightarrow F_{f}\left(t_{\varepsilon}\right) \geqq 1-\varepsilon$. Thus

$$
\lim \sup s_{n}\left(G \sim K_{t_{\varepsilon}}\right) \leqq \lim s_{n}\left(\left\{x: f(x)>t_{\varepsilon}\right\}\right) \leqq-\log (1-\varepsilon) \sim \varepsilon .
$$

This is a contradiction and so $\left\{s_{n}\right\}$ is tight.

If $f$ is a continuous function with $f(0)=0$, it is easy to verify that $\int f d s_{n} \rightarrow I(f)$ where $I$ is a positive linear functional. Using an argument as in the Riesz representation theorem, $I$ can be expressed in the form $I(f)=\int f d s$ for some regular Borel measure $s$.

The converse half of the theorem is easy to prove, and we will leave it to the reader.

9. Examples. We will now give two numerical examples to illustrate various features of the preceding development.

9.1. Probability theory on the Klein 4-group. We will now discuss limit laws for the Klein 4-group, $Z_{2} \times Z_{2}$ where $Z_{2}$ is the 2 element group with the discrete topology.

It should be interesting to the reader who is acquainted with the theory of sums of independent random variables on the real line to compare some of the facts illustrated here with the corresponding facts for the theory on the real line. For example, if $\left\{m_{n}\right\}$ and $m$ are measures on the real line, if $m_{n} \Rightarrow 1$ and $m_{n}^{* n} \Rightarrow m$, then $n m_{n} \stackrel{0}{\Rightarrow} s$ for some Borel measure $s$ which has finite mass off any neighborhood of the origin. Furthermore, $s$ is completely specified (except at the origin) by $m$. In contrast to this, our examples on $Z_{2} \times Z_{2}$ will show that if $m_{n} \Rightarrow 1$ and $m_{n}^{* n} \Rightarrow m$, then

(1) the limit $s: n m_{n} \stackrel{0}{\Rightarrow} s$ may exist but have infinite mass off some neighborhood of 1 ,

(2) the limit $s$ may not exist as a unique limit,

(3) several distinct limit measures $s$ may exist for the same measure $m$, corresponding to different choices of the measures $m_{n}$. 
9.1.1. The character group of $Z_{2} \times Z_{2}$. The character group is easily calculated $[6, \S 23.27]$. We will use positional notation on a $2 \times 2$ matrix to denote the group, character group, and functions. Thus if one writes $\{1, x\}$ for the elements of $Z_{2}$, then the elements of $Z_{2} \times Z_{2}$ are

$$
Z_{2} \times Z_{2}=\left(\begin{array}{ll}
1 & a \\
b & c
\end{array}\right)
$$

where $1=(1,1), a=(1, x), b=(x, 1)$, and $c=(x, x)$. The character group is

$$
\left(Z_{2} \times Z_{2}\right)^{\wedge}=\left(\begin{array}{ll}
\hat{1} & \hat{a} \\
\hat{b} & \hat{c}
\end{array}\right)
$$

where

$$
\hat{1}=\left(\begin{array}{ll}
1 & 1 \\
1 & 1
\end{array}\right), \quad \hat{a}=\left(\begin{array}{ll}
1 & -1 \\
1 & -1
\end{array}\right), \quad \hat{b}=\left(\begin{array}{rr}
1 & 1 \\
-1 & -1
\end{array}\right), \quad \hat{c}=\left(\begin{array}{rr}
1 & -1 \\
-1 & 1
\end{array}\right) .
$$

9.1.2. Infinitely divisible laws. Since $x=x^{-1}$ for any element, all measures are symmetric. A simple computation shows that it is possible to compute the Fourier transform of a measure and the measure of a Fourier transform as follows:

$$
\begin{array}{cc}
m=\left(\begin{array}{ll}
p & q \\
r & s
\end{array}\right) & \hat{m}=\left(\begin{array}{ll}
1 & \beta \\
\gamma & \delta
\end{array}\right) \\
1=p+q+r+s, & 4 p=1+\beta+\gamma+\delta, \\
\beta=p-q+r-s, & 4 q=1-\beta+\gamma-\delta, \\
\gamma=p+q-r-s, & 4 r=1+\beta-\gamma-\delta, \\
\delta=p-q-r+s, & 4 s=1-\beta-\gamma+\delta .
\end{array}
$$

Note that $\beta, \gamma$, and $\delta$ are real valued.

If $m$ is infinitely divisible, then $m=m_{n}^{* n}$ for each integer $n$, and

$$
\hat{m}=\left(\begin{array}{ll}
1 & \beta_{n}^{n} \\
\gamma_{n}^{n} & \delta_{n}^{n}
\end{array}\right)
$$

Since the entries in this matrix are real, and $n$ can be even or odd, it follows that $\beta, \gamma$, and $\delta$ are nonnegative. In fact, the infinitely divisible measures are precisely those measures which have Fourier transforms of the form

$$
\hat{m}=\left(\begin{array}{ll}
1 & e \\
f & g
\end{array}\right), \quad e, f, g \geqq 0 \text { and } \quad 1+e \geqq f+g, 1+f \geqq e+g, 1+g \geqq e+f .
$$

We remark parenthetically that the idempotent measure whose Fourier transform is

$$
\hat{m}=\left(\begin{array}{ll}
1 & 0 \\
0 & 1
\end{array}\right)
$$

is not infinitely divisible in the sense of Grenander, [7], even though it is, as we shall see, a possible limit law for infinitesimal sequences of symmetric measures. 
We leave it to the reader to check that for this measure one cannot find a sequence $\left\{m_{n}\right\}$ with $m_{n} \Rightarrow 1$ and $m_{n}^{* n}=m$.

9.1.3. An illustrative example. We will now give a simple example which turns on the fact that if $0<x<1$ and $x^{1 / n} \sim 1$, then $\log x \sim-n\left(1-x^{1 / n}\right)$. Let $\left\{a_{n}\right\}$ be a sequence of positive numbers such that $a_{n} \rightarrow 0$ and $a_{n}^{1 / n} \rightarrow 1$. Let $0<\delta \leqq 1$. Define $m_{n}$ and $m$ as follows:

$$
\begin{aligned}
\hat{m} & =\left(\begin{array}{ll}
1 & 0 \\
0 & \delta
\end{array}\right), & m & =\left(\begin{array}{cc}
(1+\delta) / 4 & (1-\delta) / 4 \\
(1-\delta) / 4 & (1+\delta) / 4
\end{array}\right), \\
\hat{m}_{n} & =\left(\begin{array}{cc}
1 & a_{n}^{1 / n} \\
a_{n}^{1 / n} & \delta^{1 / n}
\end{array}\right), & m_{n} & =\left(\begin{array}{cc}
\left(1+\delta^{1 / n}+2 a_{n}^{1 / n}\right) / 4 & \left(1-\delta^{1 / n}\right) / 4 \\
\left(1-\delta^{1 / n}\right) / 4 & \left(1+\delta^{1 / n}-2 a_{n}^{1 / n}\right) / 4
\end{array}\right) .
\end{aligned}
$$

All entries of $m_{n}$ are nonnegative provided $a_{n} \leqq \delta$. By construction, $m_{n} \Rightarrow 1$ and $m_{n}^{* n} \Rightarrow m$. We can compute the limit of $n m_{n}$ with the result

$$
n m_{n} \rightarrow\left(\begin{array}{cc}
\infty & -(\log \delta) / 4 \\
-(\log \delta) / 4 & \infty
\end{array}\right)=s .
$$

Thus we see that $n m_{n} \stackrel{0}{\Rightarrow} s$ where $s$ has infinite mass off the identity (an open set). With $\delta=1$, the limit is the idempotent measure mentioned above. Applying Theorem 8.1 we find that with probability tending to 1 , some of the random variables $\xi_{n j}$ assume values other than 1 , and the limiting probability that the random variables in a row of the infinitesimal array all fall in the set $\{1, c\}$ is $\sqrt{ } \delta$.

Using the same notation as before, define a new sequence of measures $m_{n}^{\prime}$ with Fourier transforms

$$
\hat{m}_{n}^{\prime}=\left(\begin{array}{cc}
1 & \left(a_{n} \delta\right)^{1 / n} \\
a_{n}^{1 / n} & \delta^{1 / n}
\end{array}\right) .
$$

Then $m_{n}^{\prime} \Rightarrow 1$ and $m_{n}^{\prime * n} \Rightarrow m$. However, this time we obtain for the limit of $n m_{n}^{\prime}$

$$
n m_{n}^{\prime} \rightarrow\left(\begin{array}{cc}
\infty & -(\log \delta) / 2 \\
0 & \infty
\end{array}\right)=s^{\prime} .
$$

Thus for a given limit measure $m$ it is possible to find infinitesimal arrays which converge to that limit measure, but which circle converge to different measures, and so the limit $s$ is not uniquely determined by $m$. An easy step from this is the observation that if the sequences $\left\{m_{n}\right\}$ and $\left\{m_{n}^{\prime}\right\}$ are mixed in some appropriate fashion, then it is possible to obtain an array which does not circle converge at all (because it oscillates between the limits $s$ and $s^{\prime}$ ).

9.2. Probability on the circle group. We will give examples of some expected results as they appear in the circle group $T$.

The character group of $T$ is isomorphic to $Z$, the group of integers. Thus $T^{\wedge}$ is generated by a single element. $T$ is a special example of a locally compact abelian group in two important ways: $T$ is metrizable and the index set $I$ of the special function $g(x, \gamma)$ is finite. One might also comment that since $T^{\wedge}$ is discrete, all functions on $T^{\wedge}$ are continuous. 
We will write elements of $T$ in the form $x=e^{i y}:-\pi \leqq y<\pi$ and elements of $T^{\wedge}$ in the form $\gamma_{n}=e^{i n y}: n \in Z$. The special function $g(x, \gamma)$ is defined by $g(x, \gamma)$ $=g(x) h(\gamma)$ where $h\left(e^{i n y}\right)=n$ and $g(x)$ is a bounded continuous function on $T$ such that $g\left(e^{i y}\right)=y$ for $y$ in $[-1,1]$. We will now illustrate various facts about the circle group with some examples.

Let $m_{n}$ be the Haar measure of $T$ restricted to $\left\{x=e^{i y}:|y| \leqq \pi / n\right\}$ and normalized so that $m_{n}(T)=1$. If $\theta(n)$ is any increasing function of $n, \theta(n) m_{n} \stackrel{0}{\Rightarrow} 0$. The integral $\int g^{2}(x) d s_{n}(x)$ which appears in Theorem 4.1 has the following form with $s_{n}=\theta(n) m_{n}$ :

$$
\int g^{2}(x) d s_{n}(x)=\pi^{2} \theta(n) / 3 n^{2}
$$

The measure corresponding to the $\theta(n)$-fold convolution of $m_{n}$ is obtained from the Fourier transform of $m_{n}$ :

$$
\begin{aligned}
\hat{m}_{n}\left(\gamma_{m}\right) & =\frac{n}{m \pi} \sin \frac{m \pi}{n}, \\
{\left[\hat{m}_{n}\left(\gamma_{m}\right)\right]^{\theta(n)} } & =\exp \left[\theta(n) \log \left[\frac{n}{m \pi} \sin \frac{m \pi}{n}\right]\right] .
\end{aligned}
$$

As $n \rightarrow \infty, n / m \pi \rightarrow \infty$ and $(n / m \pi) \sin (m \pi / n) \rightarrow 1$ for each fixed $m$. Hence

$$
\lim _{n}\left[\hat{m}_{n}\left(\gamma_{m}\right)\right]^{\theta(n)}=\lim _{n} \exp \left[-m^{2} \pi^{2} \theta(n) / 6 n^{2}\right] .
$$

From these computations we can summarize several cases of weak convergence. The following table lists cases of interest. For the table we have $m_{n}^{* k_{n}} \Rightarrow m$, $k_{n} m_{n} \stackrel{0}{\Rightarrow} s$ and $\int x^{2} d s_{n} \rightarrow c$.

\begin{tabular}{c|c|c|c}
$k_{n}$ & $m$ & $s$ & $c$ \\
\hline$n$ & 1 & 0 & 0 \\
$n^{2}$ & (a) & 0 & $\pi^{2} / 3$ \\
$n^{j}, j>2$ & (b) & 0 & $\infty$
\end{tabular}

(a) is the measure such that $\hat{m}\left(\gamma_{j}\right)=\exp \left[-j^{2} \pi^{2} / 6\right]$,

(b) is the Haar measure of $T$.

For a second example, let $m_{n}=\lambda / n^{2}+\left(1-1 / n^{2}\right) 1$ where $\lambda$ is the Haar measure of $T$. Then the table corresponding to the above is

\begin{tabular}{c|c|c|c}
$k_{n}$ & $m$ & $s$ & $c$ \\
\hline$n$ & 1 & 0 & 0 \\
$n^{2}$ & $(\mathrm{c})$ & $\lambda$ & $\pi^{2} / 3$ \\
$n^{j}, j>2$ & $\lambda$ & $\infty$ & $\infty$
\end{tabular}

(c) for this measure, $\hat{m}\left(\gamma_{j}\right)=e^{-1}$ except for $j=0$.

One conclusion which can be drawn from these examples on the Klein 4-group and the circle group is the fact that the two conditions for the limit law $m$ to have 
an idempotent factor, given in Theorem 4.1, are independent. Our examples on the Klein 4-group (discrete, and hence $g(x, \gamma) \equiv 0$ ) show that (ii) need not hold and the examples on the circle group show that (i) need not hold in order for the limit to have an idempotent factor.

\section{REFERENCES}

1. K. R. Parthasarathy, Probability measures on metric spaces, Probability and Math. Statist., no. 3, Academic Press, New York, 1967. MR 37 \#2271.

2. B. V. Gnedenko and A. N. Kolmogorov, Limit distributions for sums of independent random variables, GITTL, Moscow, 1949; English transl., Addison-Wesley, Reading, Mass., 1954. MR 12, 839; MR 16, 52.

3. $\mathrm{Ju}$. V. Prohorov, Convergence of random processes and limit theorems in probability theory, Teor. Verojatnost. i Primenen. 1 (1956), 177-238= Theory Probability Appl. 1 (1956), 157-214. MR 18, 943.

4. K. R. Parthasarathy, R. Ranga Rao and S. R. S. Varahan, On the category of indecomposable distributions on topological groups, Trans. Amer. Math. Soc. 102 (1962), 200-217. MR 27 \#3010.

5. D. C. Bossard, Probability on locally compact abelian groups: Sums of independent random variables, Thesis, Dartmouth College, Hanover, N. H., 1967.

6. E. Hewitt and K. A. Ross, Abstract harmonic analysis. Vol. I: Structure of topological groups. Integration theory, group representations, Die Grundlehren der math. Wissenschaften, Band 115, Academic Press, New York; Springer-Verlag, Berlin, 1963. MR 28 \#158.

7. U. Grenander, Probabilities on algebraic structures, Wiley, New York; Almqvist \& Wiksell, Stockholm, 1963. MR 34 \#6810.

\section{Dartmouth College,} HaNOVER, New HaMpshire 03755

Daniel H. Wagner, Associates, Paoli, Pennsylvania 19301 